\title{
A texture segmentation prototype for industrial inspection applications based on fuzzy grammar
}

\author{
Manuel Ferreira, Cristina Santos and foao Monteiro \\ Department of Industrial Electronics, University of Minho, Guimaraes, Portugal
}

\begin{abstract}
Purpose - The purpose of this paper is to propose a set of techniques, in the domain of texture analysis, dedicated to the classification of industrial textures. One of the main purposes was to deal with a high diversity of textures, including structural and highly random patterns.

Design/methodology/approach - The global system includes a texture segmentation phase and a classification phase. The approach for image texture segmentation is based on features extracted from wavelets transform, fuzzy spectrum and interaction maps. The classification architecture uses a fuzzy grammar inference system.

Findings - The classifier uses the aggregation of features from the several segmentation techniques, resulting in high flexibility concerning the diversity of industrial textures. The resulted system allows on-line learning of new textures. This approach avoids the need for a global re-learning of the all textures each time a new texture is presented to the system.

Practical implications - These achievements demonstrate the practical value of the system, as it can be applied to different industrial sectors for quality control operations.

Originality/value - The global approach was integrated in a cork vision system, leading to an industrial prototype that has already been tested. Similarly, it was tested in a textile machine, for a specific fabric inspection, and gave results that corroborate the diversity of possible applications. The segmentation procedure reveals good performance that is indicated by high classification rates, revealing good perspectives for full industrialization.
\end{abstract}

Keywords Textiles, Fabric inspection, Textile technology, Quality control, Fuzzy control

Paper type Research paper

\section{Introduction}

In several industrial sectors, like fabric, wood planks, and cork parquet production, one of the most important visual properties that must be subject to quality control is the texture of raw materials and products. However, for industrial inspection applications with highly texture environments or objects, and where the light conditions are not stable or its interaction with the objects produces shadows, texture becomes somehow a difficult cue to analyze.

The most common texture segmentation techniques tend to be computationally intensive. Additionally, they use classification methods that require a time expensive off-line learning phase and that demand a great amount of samples to perform learning. For these reasons, such approaches are not consistently used in industrial inspection applications. There is only a few number of available commercial solutions and all present several limitations concerning the

The current issue and full text archive of this journal is available at www.emeraldinsight.com/0260-2288.htm

Sensor Review

29/2 (2009) 163-173

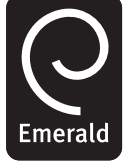

(C) Emerald Group Publishing Limited [ISSN 0260-2288]

[DOI 10.1108/02602280910936273] flexibility to learn new textures and the number of texture to be learned.

The major motivation for this work was the development of a generic laboratory prototype with the ability to:

- Integrate information of several texture techniques.

- To be flexible concerning the type and the number of textures.

This paper describes the approach developed for texture segmentation based on gray level images. This approach reflect on two major constraints: the processing time and the concern about the multiplicity of texture patterns. Figure 1 presents examples of the diversity of textures that must be analyzed.

The major problem in texture segmentation is the extraction of texture features for the classification procedure. The classifier must deal with the following constraints:

- High diversity of texture objects.

- The learning phase must run with a unique sample of each type of texture.

There is no formal or complete definition of texture, as it still depends on the technological and scientific area. Even in the same area, depending on the application and on the researcher, it

The authors would like to thank the anonymous reviewers for the valuable comments and suggestions. 
Figure 1 Illustration of the diversity of texture to be analyzed

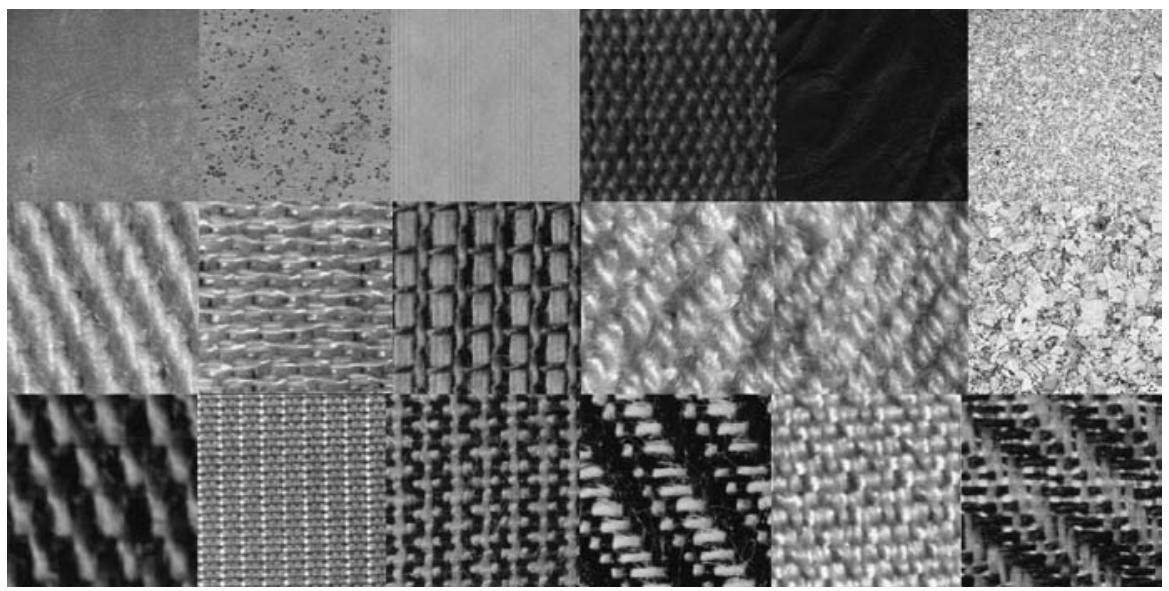

may has different meanings (Haralick and Shapiro, 1992; Russ, 1995; Williams, 1999; Pratt, 2001; Bharati et al., 2004). In the context of image analysis, for instance accordingly with (Gonzalez and Woods, 1992; Haralick and Shapiro, 1992), texture is a descriptor from which it is possible to infer properties, like smoothness, coarseness, depth and regularity. (Ballard and Brown, 1982) presents texture as a structure composed of a large number of similar elements - texel - disposed with a specific arrangement. Nevertheless, texture is a concept that depends on the observation scale (Haralick and Shapiro, 1992; Heeger and Bergen, 1995; Russ, 1995; Randen, 1997; Livens, 1998; Wouwer, 1998; Pratt, 2001). Considering only monochromatic images, texture can be viewed as an image attribute representing the spatial distribution of the gray levels of the pixels.

The classical techniques for analyzing texture can be classified as statistical, structural, and spectral (Ballard and Brown, 1982; Haralick and Shapiro, 1992; Pratt, 2001). New ones have been more recently under research, such as features extracted from the wavelets transform (Laine and Fan, 1993; Unser, 1995; Livens, 1998; Wouwer, 1998; Wouwer et al., 1998; Latif-Amet et al., 2000; Tay and DeBrunner, 2002; Liapis et al., 2004; JafariKhouzani and Soltanian-Zadeh, 2005), Gabor filters (Bovik et al., 1990; Teuner et al., 1995; Williams, 1999; Pratt, 2001; Paragios and Deriche, 2002; Ma et al., 2003), fractals (Chaudhuri and Sarkar, 1995; Russ, 1995; Zhang et al., 1995; Neary, 2000; Ducher et al., 2004; Huang et al., 2006), mathematical morphology (Gonzalez and Woods, 1992; Haralick and Shapiro, 1992; Mirmehdi et al., 1996; Moraes, 1996), Markov random fields (Cross and Jain, 1983; Williams, 1999; Tseng and Lai, 1999; Fan and Xia, 2003; Clausi and Yue, 2004), feature based interaction maps (FBIM) of pixels (Chetverikov, 1995, 1999) and fuzzy logic techniques (Lee et al., 1998).

The segmentation techniques based on the wavelet transform, Gabor filters, fractals, and FBIM, deserve special attention, specifically because of their similarity with the human vision system, which processes visual information in a multiscale manner. In this way, they incorporate the notion of scale, allowing the identification of micro and macro textures.

Regarding the classifiers and recognizers, there are also different types of approach. The most common solutions use recognizers based on the calculus of metrics like Euclidean, Minkowsky and Mahalanobis distance measures. Others use multivariate statistical approaches (based on PCA and PLS), Hidden Markov Models, and soft-computing techniques (neural, fuzzy logic, and neurofuzzy networks) (Duda and Hart, 1973; Carpenter and Grossberg, 1992; Tzanakou, 2000; Costa and Cesar, 2001; Bennamoun and Mamic, 2002; Looney, 2002; Perry et al., 2002; Bharati et al., 2004; Chen and Chu, 2005). However, these recognizers, even the unsupervised, demand a great amount of samples from the population to perform the learning procedure.

The validation of the results of texture segmentation is normally accomplished using the Brodatz (1966) and VisTEX (1997) databases. An important observation regarding the works mentioned above is the fact that the validation is made using a restrict number of textures (typically 5-40 images). Other conclusion is that different authors use different texture images, which increase the difficulty to compare the performance of the segmentation techniques.

Texture analysis continues to play an important role in many application areas, with its use increasing with the availability of faster processors, such as medical imaging, biometrics, remote sensing, tracking objects in robotics, image retrieval, and industrial inspection; its tasks are mainly classification and segmentation (Zhang and Tan, 2002; Karkanis et al., 2003; Tsai and Huang, 2003; Clausi and Yue, 2004; Cui et al., 2004; Ducher et al., 2004; Liu et al., 2004; Munzenmayer et al., 2005; Huang et al., 2006; PratsMontalban and Ferrer, 2007). The majority of the authors use only one technique to describe the texture. However, due to the diversity of textures present in industrial sectors, this seems to be inadequate for applications that must have high level of flexibility in order to be generically applied.

The present work combine techniques in such a way that texture segmentation is based on features extracted from the wavelet transform, fuzzy spectrum and FBIM, using a fuzzy grammar as classifier.

\section{System architecture}

The texture segmentation procedure is divided in two phases (Figure 2): the learning and the execution phases. In the learning phase, a texture is manually selected and a fuzzy rule is generated and stored in the database. In the execution phase, the texture under analysis is submitted to each fuzzy rule stored in the database and a texture identification is performed. The system is capable of automatically starting a learning phase in case an unknown texture is shown to the system. 
Figure 2 Architecture of the processing system

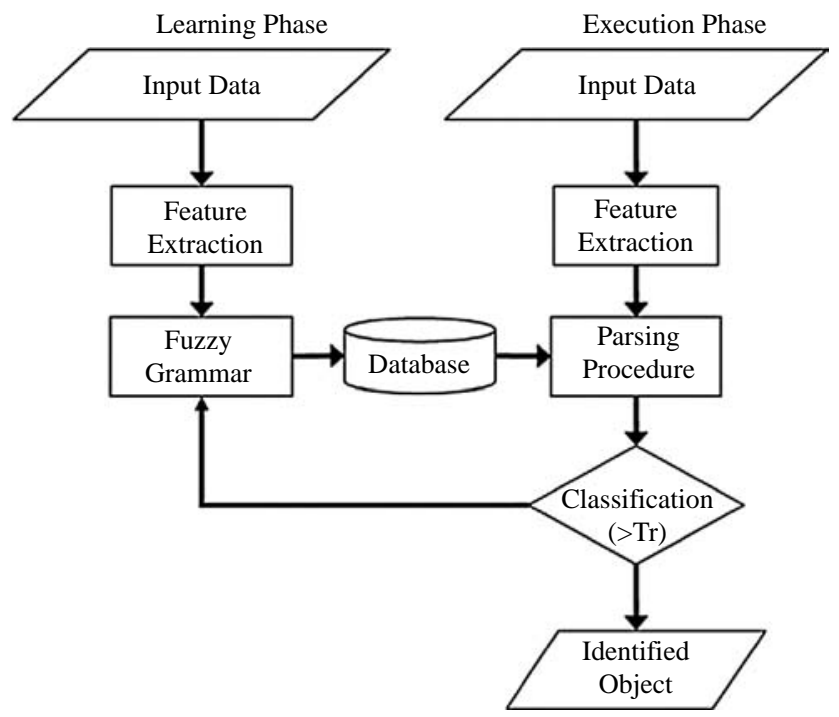

The feature extraction module (Figure 2) extracts the feature vector that best describes each texture - features from the wavelet transform, fuzzy spectrum and from FBIM.

The fuzzy grammar module uses the extracted feature vector to generate the fuzzy rule that describes the texture.

In the execution phase, the extracted feature vector is submitted to a parsing procedure module. The vector is submitted to each fuzzy rule stored on the database, and a response value in the interval $[0,1]$, reflecting the grade of membership of the texture, is obtained. A DLL was created to encapsulate the parsing procedure, which was developed with the compilers yacc and lex (Bumble-Bee, 2008).

Finally, the Classification module uses the output of the parsing and verifies which rule produces a value higher than a pre-defined threshold. If a new texture is presented to the system during the execution phase, the response value is low, and, consequently, a learning phase is automatically initiated to generate an appropriate fuzzy rule for that texture.

\section{Feature extraction module}

Tests performed with different types of materials had indicated that the best compromise between high texture diversity and classification rate is achieved with the wavelet transform, fuzzy spectrum and FBIM techniques. Thus, these techniques were chosen to integrate the generic prototype. The fuzzy spectrum is used by its capability to describe the uniformity of the surfaces. FBIM is a multiscale approach and is used due to its ability to infer the orientation and symmetry of the patterns. The wavelets transform is a multiscale analysis and it allows obtaining directional properties of the texture.

\subsection{Wavelets transform}

Theoretical fundamentals of the wavelet transform applied to signal analysis are discussed in (Benedetto and Frazier, 1994). The wavelet transform in this domain introduces the concept of a time window variable with frequency. Signal events with high frequency are analyzed with a timing resolution higher than the ones with lower frequency. Figure 3 summarizes the decomposition of a generic signal using the discrete wavelet
Figure 3 Signal decomposition using DWT

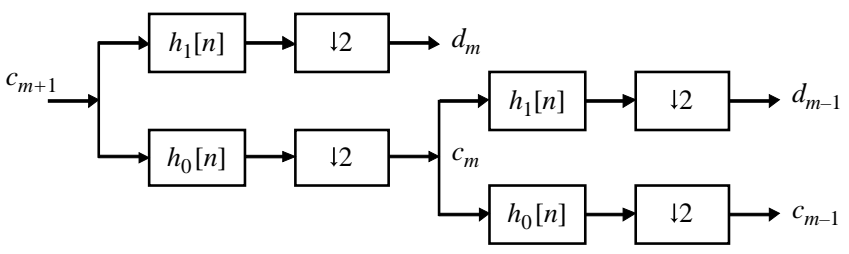

transform (DWT). It can be viewed as the application of lowpass filters $\left(h_{0}[n]\right)$ and high-pass filters $\left(h_{1}[n]\right)$, followed by a sub-sampling.

To perform the wavelet transform, in the context of image processing, it is necessary to employ a two-dimensional DWT. As illustrated in Figure 4(a), an approximation coefficient, at level $m+1 \quad\left(V_{m+1} \times V_{m+1}\right)$, is decomposed in four components: the approximation coefficient at level $m$ $\left(V_{m} \times V_{m}\right)$ and the details at level $m$ in three orientation coefficients: horizontal $\left(V_{m} \times W_{m}\right)$, vertical $\left(W_{m} \times V_{m}\right)$ and diagonal $\left(W_{m} \times W_{m}\right)$. These last three components are the detail images used to construct the feature vectors. Figure 4(b) shows a cork textured image and the correspondent wavelet transforms. There are several types of wavelets functions that can be used in texture analysis. However, the type of wavelet function does not produce relevant changes in the analysis.

This application uses three levels for the wavelet transform, which results in a total of nine detail images. The feature vector consists of features extracted from the detail images at each decomposition level. The extracted features are the following parameters: mean $(M)$, standard deviation (SD), contrast between adjacent - next neighbour - pixels in vertical

Figure 4 Wavelet decomposition of an image: (a) final image obtained by the sub-spaces $V_{-i} \times V_{-i}, V_{-i} \times W_{-i}, W_{-i} \times V_{-i}, W_{-i} \times W_{-i}$ with $i=1,2,3$; (b) image of a textured object (up) and the corresponding wavelet transform (down) with three levels of decomposition

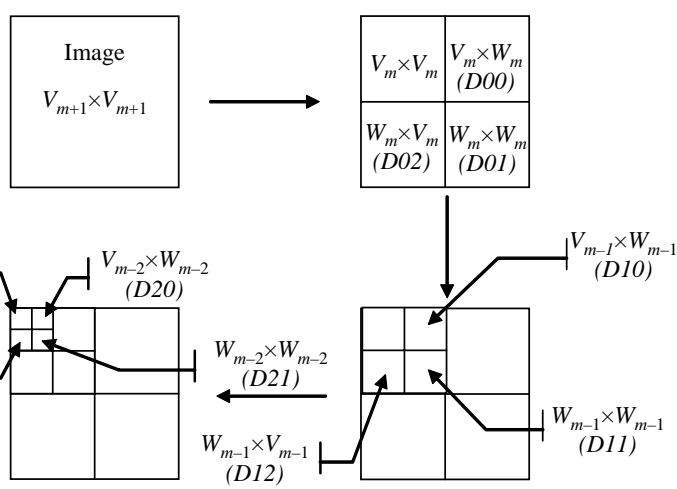

(a)
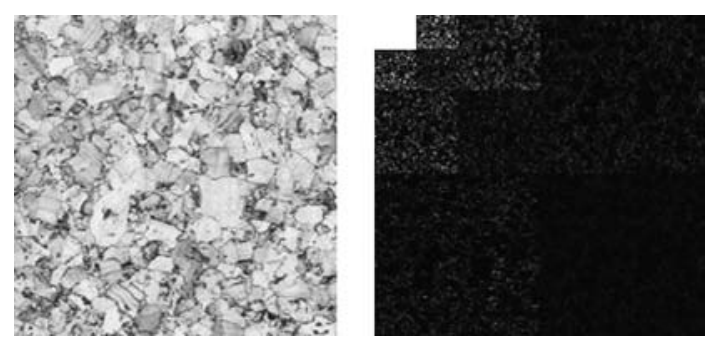

(b) 
(CBNNV) and horizontal (CBNNH) directions and contrast between alternated - alternated neighbour - pixels in vertical (CBANV) and horizontal (CBANH) directions ((1)-(6)).

Since the classifier is based on a fuzzy inference system, it implies that the magnitude of each element of the feature vector must be in the interval $[0,1]$ and, thus, a normalization of each feature element is required (7). The feature vector, FVW, consists of six features for each detail image $(6 \times 9=54$ features $): \quad \mathrm{FV}=\left[\mu_{M i j}, \quad \mu_{\mathrm{SD} i j}, \mu_{\mathrm{CBNNV} i j}\right.$, $\left.\mu_{\mathrm{CBNNH} i j}, \mu_{\mathrm{CBANV} i j}, \mu_{\mathrm{CBANH} i j}\right]$, with $i=0,1,2 ; j=0,1,2$.

$$
\begin{gathered}
M=\frac{1}{N} \sum_{i=0}^{N-1} I(i) \\
\mathrm{SD}=\sqrt{\frac{1}{N} \sum_{i=0}^{N-1}(I(i)-M)^{2}} \\
\mathrm{CBNNH}=\frac{1}{N-1} \sum_{l=0}^{N_{l}-1} \sum_{c=0}^{N_{c}-1}\left|I\left(l \times N_{c}+c+1\right)-I\left(l \times N_{c}+c\right)\right| \\
\mathrm{CBNNV}=\frac{1}{N-1} \sum_{l=0}^{N_{l}-1} \sum_{c=0}^{N_{c}-1}\left|I\left((l+1) \times N_{c}+c\right)-I\left(l \times N_{c}+c\right)\right| \\
\mathrm{CBANV}=\frac{1}{N-1} \sum_{l=0}^{N_{l}-1} \sum_{c=0}^{N_{l}-1}\left|I\left((l+2) \times N_{c}+c\right)-I\left(l \times N_{c}+c\right)\right| \\
\mu_{F}=F / 255 \quad \mid \sum_{c}-1 \\
F \in\{M, \mathrm{SD}, \mathrm{CBNNV}, \mathrm{CBNNH}, \mathrm{CBANV}, \mathrm{CBANH}\},
\end{gathered}
$$

where $I$ is the image, $N$ is the number of pixels in the image and $N_{c}$ and $N_{l}$ are the number of columns and lines in the image, respectively.

\subsection{FBIM - feature base interaction maps}

This technique, proposed by Chetverikov (1995), has higher efficiency for anisotropic textures and is based on the extraction of the texture primitive dimension: orientation and symmetry. The interaction between pairs of pixels leads to the definition of two interaction maps:

1 MPL (polar interaction map).

2 SPL (symmetry interaction map).

In Chetverikov (1995), the author considers the following:

- The interaction between pairs of pixels gives important structural information.

- The interaction between pairs of pixels at short range and long range is relevant.

- Higher angular resolution is possible and relevant.

- Structural information can be obtained by the MPL map.

- Texture orientation can be obtained by the SPL map.

The MPL consists on a polar representation of the feature extracted from extended gray-level difference histogram $(\mathrm{EGLDH})$, which is obtained by the difference of the gray levels of a pair of pixels (Figure 5). The procedure to obtain the structural information and the texture orientation includes the following:

- For a set of angles $\left(N_{a}\right)$ and a set of distances between pixels $\left(N_{d}\right)$ the value of $M\left(\alpha_{i}, d_{j}\right)$ (Figure 5$)$ is obtained,
Figure 5 Extended gray-level difference histogram

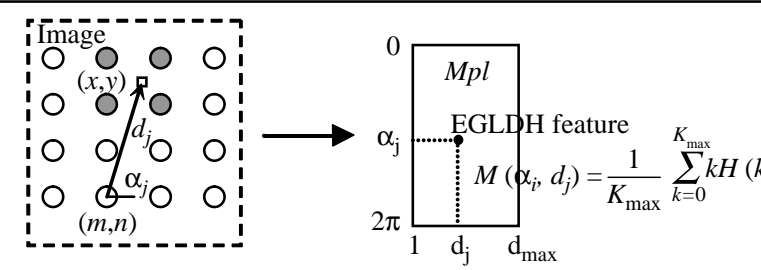

$(m, n)$ - pixel under analisys;

$\mathrm{I}(\mathrm{m}, \mathrm{n})$ - pixel gray level

$(x, y)$-image point specified by vector $v=(\alpha, d)$;

$\mathrm{I}(\mathrm{x}, \mathrm{y})$-pixel gray level obtained by neighbourhood interpolation

$H\left(k ; \alpha_{i}, d_{j}\right)=\frac{\left|\left\{(m, n),(x, y): m \in I_{1}, n \in I_{2},|I(m, n)-I(x, y)|=k\right\}\right|}{\left|\left\{(m, n),(x, y): m \in I_{1}, n \in I_{2}\right\}\right|}$

$k \in\left[0, k_{\max }\right]$,

- $\quad k_{\max }=N g-1$,

- $\quad N g$ number of gray levels,

- $\quad x=d \cos \alpha$ and $x=d \sin \alpha$

considering $\alpha_{i} \in[0,2 \pi] ; i=0,1, \ldots N_{a-1} ; d_{j} \in\left[1, d_{\max }\right]$ where $d_{\max }$ is the maximum interaction distance and $j=0,1, \ldots N_{d-1}$.

- The polar interaction map is defined has: $M_{\mathrm{pl}}(i, j)=M\left(\alpha_{i}, d_{j}\right)$.

- The symmetry indicatives, $S^{j}(i)$, are defined accordingly with (8). Where $\mathbf{A}^{j}(i)$ is the circular vector given by $\mathbf{A}^{j}(i)=M_{\mathrm{pl}}(i, j), \quad$ for $\quad j=0,1, \ldots, \quad N_{d-1} \quad$ and $i=0,1, \ldots, i_{\max }$, with $i_{\max }=N_{a-1}$. The $\gamma$ factor controls the sensibility of the symmetry and (Chetverikov, 1995) defines the typical value as $\gamma=5$.

- The symmetry map is defined has: $S_{\mathrm{pl}}(i, j)=S^{j}(i)$.

- The SPL map is divided in two distinct zones: short range and long range; for each zone a symmetry vector is defined accordingly (9) and (10); and $j$ tr is specified by (11) and (12).

$$
\begin{gathered}
S^{j}(i)=\left(\frac{2}{i_{\max }} \sum_{k=0}^{i_{\max } / 2}\left(1-\left|\frac{\mathbf{A}^{j}(i+k)-\mathbf{A}^{j}(i-k)}{\mathbf{A}^{j}(i+k)+\mathbf{A}^{j}(i-k)}\right|\right)\right)^{\gamma} \\
\mathbf{Z s r}(i)=S 0, j \operatorname{tr}(i) \\
\mathbf{Z l r}(i)=\operatorname{Sjtr}+1, N_{d-1} \\
D(j t r)=\max _{j}\{D(j)\} \\
D(j)=\sum_{i=0}^{N_{a-1}}\left|S_{\mathrm{pl}}(i, j-\delta)-S_{\mathrm{pl}}(i, j+\delta)\right|
\end{gathered}
$$

\subsection{Fuzzy spectrum}

This technique, presented by Lee et al. (1998), consists on the application of a fuzzification function to the image pixels, creating a fuzzy image. This function (13) measures the uncertainty of a pixel $p$, with coordinates $(i, j)$ and gray level $I(i, j)$, belonging to a uniform surface $R$ of dimensions $W \times W$.

$$
\mu_{i j}=1-\left(\frac{|I(i, j)-\bar{I}(i, j)|}{\max _{R} I(i, j)}\right),
$$

$\bar{I}(i, j)$ is the defined by $(14), \max _{R} I(i, j)$ is the maximum intensity in $R$. 


$$
\bar{I}(i, j)=\frac{1}{W \times W-1} \sum_{m, n \neq i, j}^{R} I(m, n)
$$

If a pixel $p$ belongs to a uniform surface, the fuzzification function gives values near 1 , and, on the opposite situation the function gives values extended through the interval between 0 and 1. Figure 6 shows feature vectors $\mathbf{Z l r}$ and Zsr.

The fuzzy spectrum can be seeing as the occurrence values of the fuzzy image. Figure 7 shows the fuzzy spectrum for two cork images. The feature vector, FVFS, consists on a cumulative version of the fuzzy spectrum with 16 values, $\mu_{\mathrm{FS}}(n)$, with $n=16$

\subsection{Feature selection}

During the learning phase it is necessary to consider the type of texture, specifically their periodical or random aspects. Therefore, the following was settled for the learning phase:

- Initially the user chooses a window that possesses the texture to be analyzed, specifying a region of interest (ROI).

Figure 6 Feature vectors ZIr and Zsr
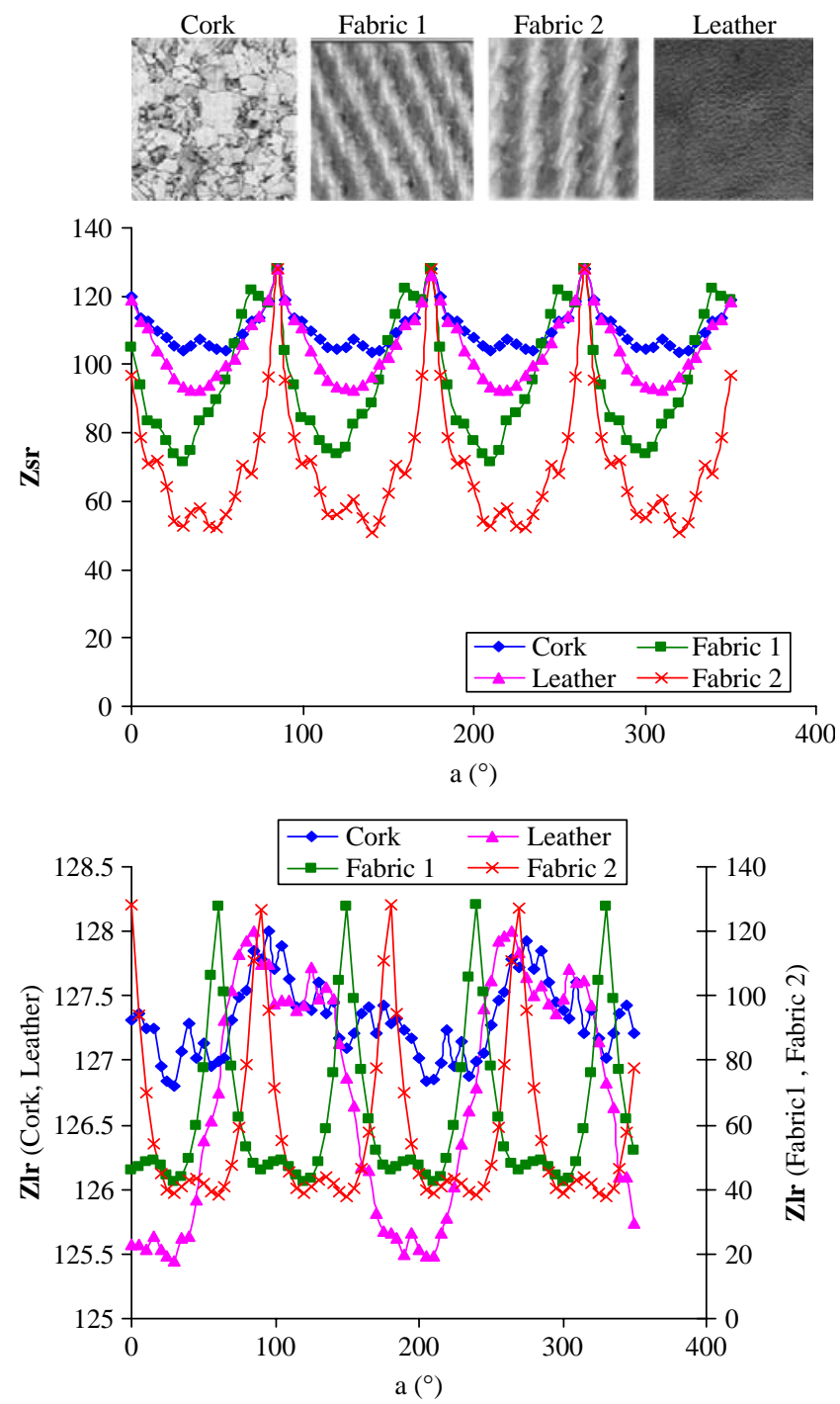

Figure 7 Fuzzy spectrum for two types of cork images: (a) fine grained cork; (b) coarse grained cork

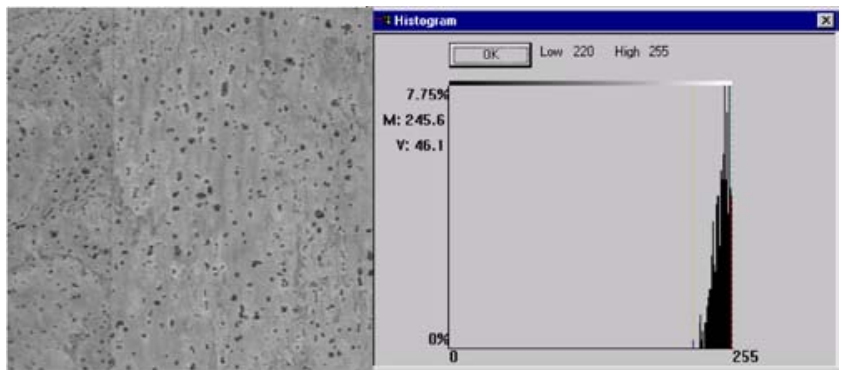

(a)

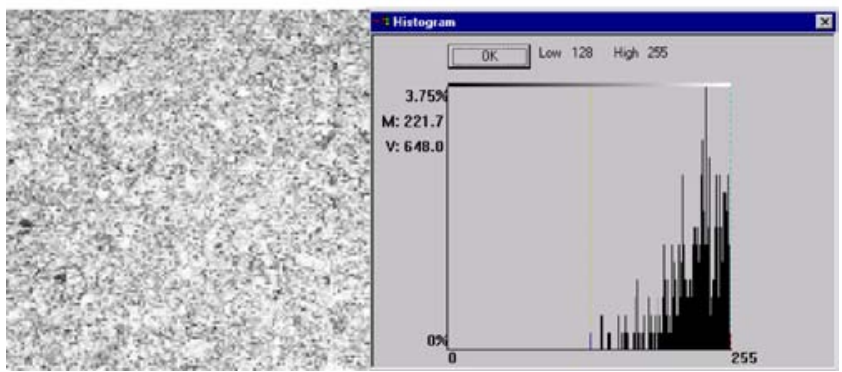

(b)

- This ROI is divided in non-overlapping windows (NOW), whose size is set by the operator (Figure 8(a)).

- For each NOW, the features FVW, FVFS, and FVFBIM are extracted. Each element of the final feature vector is the result of a feature selection procedure for each NOW.

- A fuzzy rule is created with this feature vector.

In the execution phase, the image is also divided in windows with the same size as the ones of the learning phase (NOW), but now the windows position is overlapped (Figure $8(\mathrm{~b})$ ) by $(\mathrm{d} x, \mathrm{~d} y)$, where $\mathrm{d} x, \mathrm{~d} y$ are the displacements relatively to the previous one. Such procedure ensures different grades of performance.

For all the extracted features, it is fundamental to select $n$ representative features from $N$ initials, where $n$ is much smaller than $N$. This procedure, depicted in Figure 9, enables to eliminate both the non-representative features of a particular texture and the redundant ones. Zero value and high correlation were the criteria used to eliminate features.

Figure 8 Decomposition process of the image for the application of the feature extraction block: (a) learning phase; (b) execution phase: red window $\mathrm{d} x=0, \mathrm{~d} y=0$; blue window $\mathrm{d} x=D / 2, \mathrm{~d} y=0$; green window $\mathrm{d} x=D / 2, \mathrm{~d} y=D / 2$

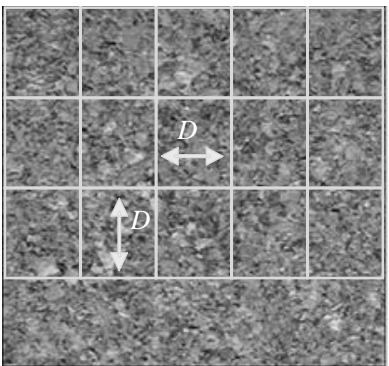

(a)

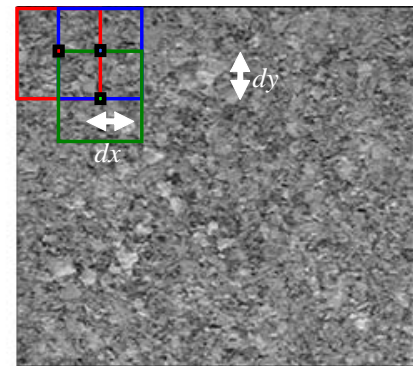

(b) 
Figure 9 Schematic representation of the procedure for feature selection

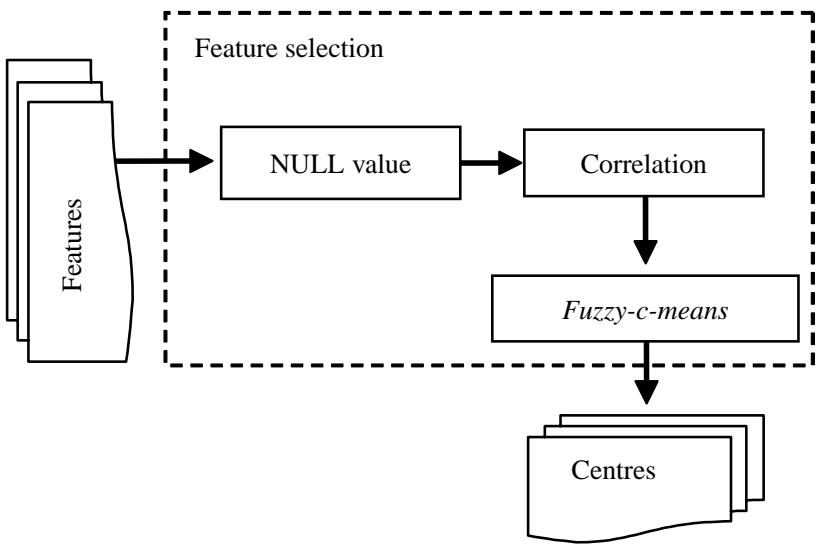

The correlation of the feature set is evaluated through fuzzy correlation (Malaviya, 1996) (15):

$$
c_{j k}=1-\frac{4 \times \sum_{i=0}^{N-1}\left(\mu_{i j}-\mu_{i k}\right)^{2}}{\sum_{i=0}^{N-1}\left(\left(2 \times \mu_{i j}-1\right)^{2}+\left(2 \times \mu_{i k}-1\right)^{2}\right)}
$$

where $N$ is the universe dimension, $\mu_{j}$ is the set of values of feature $j$ and $\mu_{k}$ is the set of values of feature $k$.

Equation (15) estimates the correlation coefficient between the values of a feature $j\left(\mu_{j}\right)$ and the set of values of the feature $k\left(\mu_{k}\right)$. If they are strongly correlated, only one is selected to be part of the final feature vector. In this work, the threshold used for the correlation coefficient was 0.9 , value that was obtained by testing the feature vectors for several textures. A lower value will increase the number of redundant features that are present on the final feature vector.

For a particular texture, in the cases where more than one value for each feature occur (most of the cases), it is necessary to select the value or the values that best describe it. This was done applying a fuzzy c-means algorithm (Bezdek and Pal, 1992; Jang et al., 1997). Several attempts, concerning the number of centres, were evaluated, resulting a good description with five centres.

Figure 10 illustrates the application of the fuzzy-c-means algorithm to the features mean and SD of the detail image D22, for the three layers of cork parquet.

\section{Fuzzy grammar module}

After the extraction of the feature vector that characterizes a texture, it is necessary to classify it according to its attributes. Specifically, the application has to deal with a high diversity of texture objects. To fulfil this constraint, the learning phase must be done with a unique sample of each type of texture.

In this work, a fuzzy system modelling approach was developed, in which a fuzzy inference system identifies the fuzzy rules representing relationships among the features extracted from the wavelet detail images. There are several approaches that generate these fuzzy rules. The most often applied are based on statistics, neural networks and genetic algorithms (Bezdek and Pal, 1992; Ivancic and Malaviya, 1998; Tzanakou, 2000). However, these methods poorly satisfy the needs of this application, specifically the possibility to learn using only a characteristic vector. Therefore, a fuzzy grammar approach was applied.
Figure 10 Fuzzy-c-means application to the features $\bar{I}$ and DP for detail image D22, for three types of cork texture

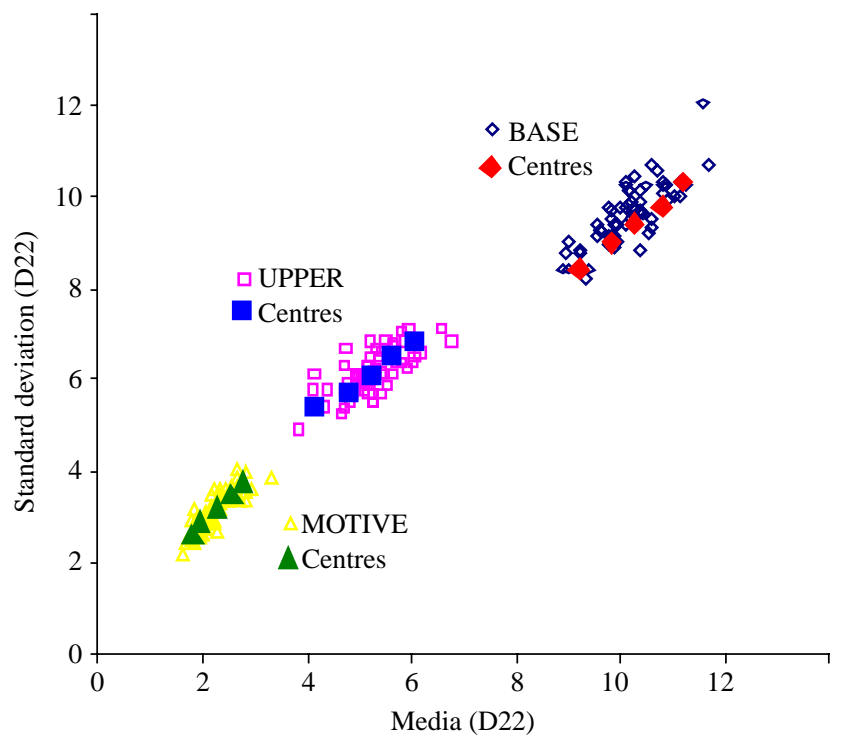

Fuzzy grammar is a pattern classification syntactic model used to represent the structural relations of patterns and that describes the syntax of the fuzzy languages, which generate the fuzzy rules. This inference system is capable of generating a fuzzy rule using only one sample of a pattern.

Herein, a brief review of some basic concepts of fuzzy grammar is presented (Bezdek and Pal, 1992; Yager and Zadeh, 1992; Malaviya, 1996). Fuzzy grammar GF is a quintuple $\mathrm{GF}=\left(V_{N}, V_{T}, P, S_{0}, \mu\right)$, in which $V_{N}$ and $V_{T}$ are finite disjoint sets of non-terminal and terminal vocabulary, respectively, such that $V=V_{N} \cup V_{T}$ is the total vocabulary of the grammar. $P$ is a finite set of production rules of the type $\alpha \rightarrow \beta$, with $\alpha \in V_{N}$ and $\beta$ is a member of the set $V^{*}$ of all strings (including the null string $\varepsilon$ ). $S_{0} \in V_{N}$ is the starting symbol. $\mu$ is the mapping of $P \rightarrow[0,1]$, such that $\mu(p)$ denotes the possibility of the current language sentence $p \in P$.

The syntax of the developed language $L(\mathrm{GF})$ includes four different steps (Malaviya, 1996):

1 The codification of the features to primitives (Table I).

2 The definition of linguistic terms - HistVar:c.

$$
\text { HistVar : } c=\prod(x, 0.2, c \times 0.1) \quad c=0 \ldots 10 .
$$

Table I Codification of features to primitives, with $i=0,1,2 ; j=0,1,2$; $n=0.71$

\begin{tabular}{ll}
\hline Feature & Primitive \\
\hline$\mu_{M i j}$ & FWDijM \\
$\mu_{\mathrm{SD} i j}$ & FWDij SD \\
$\mu_{\mathrm{CBNNV} i j}$ & FWDij CBNNV \\
$\mu_{\mathrm{CBNNH} i j}$ & FWDij CBNNH \\
$\mu_{\mathrm{CBANV} i j}$ & FWDij CBANV \\
$\mu_{\mathrm{CBANH} i j}$ & FWDij CBANH \\
$\mu_{Z s}(n)$ & FFBIMZLRn \\
$\mu_{Z 1}(n)$ & FFBIMZSRn \\
$\mu_{\mathrm{FS}}(n)$ & FFSn \\
\hline
\end{tabular}


In the membership function $\prod$ the parameter $c$ is chosen such that the eleven membership functions cover the all universe of discourse, $X$, and have disjointed maximums (Bezdek and Pal, 1992; Malaviya, 1996).

3 The definition of fuzzy modifiers (FM): "More than", "Less than" and "Between". The FM "More than" LT is defined by:

$$
\mu_{\mathrm{MT}}\langle\mathrm{LT}\rangle=\left\{\begin{array}{cc}
1 & x \geq L \\
S(x, L-\mathrm{lb}, L-\mathrm{lb} / 2, L & x<L
\end{array}\right.
$$

where $L$ is a threshold value and $l b$ is the bandwidth value of the $S$ membership function (Bezdek and Pal, 1992; Malaviya, 1996). The FM "Less than" LT is given by:

$$
\mu_{\mathrm{LT}}\langle\mathrm{LT}\rangle=\left\{\begin{array}{cc}
1 & x \leq L \\
1-S(x, L, L+\mathrm{lb} / 2, L+\mathrm{lb} & x>L
\end{array}\right.
$$

The FM "Between" $\mathrm{LT}_{1}$ e $\mathrm{LT}_{2}$, is given by:

$\mu_{B}\left\langle\mathrm{TL}_{1}\right\rangle\left\langle\mathrm{TL}_{2}\right\rangle=\left\{\begin{array}{cc}1-S\left(x, w_{1}, w_{1}+\mathrm{lb} / 2, w_{1}+\mathrm{lb}\right) & x>w_{1} \\ 1 & w_{2} \leq x \leq w_{1} \\ S\left(x, w_{2}-\mathrm{lb}, w_{2}-\mathrm{lb} / 2, w_{2}\right) & x<w_{2}\end{array}\right.$

where $w_{1}$ and $w_{2}$ are threshold values (Bezdek and Pal, 1992; Malaviya, 1996).

4 The definition of fuzzy operators (FO) which establish the relations between the linguistic terms and primitives. The following $\mathrm{FO}$ were defined:

- \&, representing the AND of two primitives. It is given by the Yager intersection.

- $>$, representing "More than" LT and is given by $\mu_{\mathrm{MT}}<\mathrm{LT}>$

- $<$, means "Less than" LT and is given by the function $\mu_{\mathrm{LT}}<\mathrm{LT}>$.

- || , describes "Between two" LT and is given by $\mu_{B}<L T_{1}><\mathrm{LT}_{2}>$.

- \#, means a "Separator between a" primitive and a LT.

- ( ), imposes a hierarchy in the rule.

For each texture technique a partial fuzzy rule is created (Figure 11). The texture is completed defined by the final rule resulting from the aggregation of the three partial rules, using Yager intersection.

Consider the texture depicted in Figure 4(b). Figure 12 illustrates the values of the eleven membership function $\int$ for the primitive FWD00M (Figure 12(a)), primitive FWD21 CBNNV (Figure 12(b)), primitive FWD22CBNNV (Figure 12(c)) and primitive FWD22CBANV (Figure 12(d)). Primitive FWD00M has non-zero degrees of membership for LT HistVar:0, LT HistVar: 1 and LT HistVar:2. The highest fuzzy value is obtained using LT HistVar:0. Thus, HistVar:0\# FWD00M is part of the fuzzy rule which characterizes this texture.

If more than one linguistic term gives fuzzy values superior to 0.75 , fuzzy modifiers like "More than", "Less than" and "Between", is applied to combine the obtained results. Accordingly, > HistVar:9\# FWD22CBNNV is part of the fuzzy rule which characterizes this texture for primitive FWD21CBNNV. For primitive FWD22CBNNV, the result will be < HistVar:1\# FWD22CBNNV and for the primitive
Figure 11 Procedure to create the final fuzzy rule

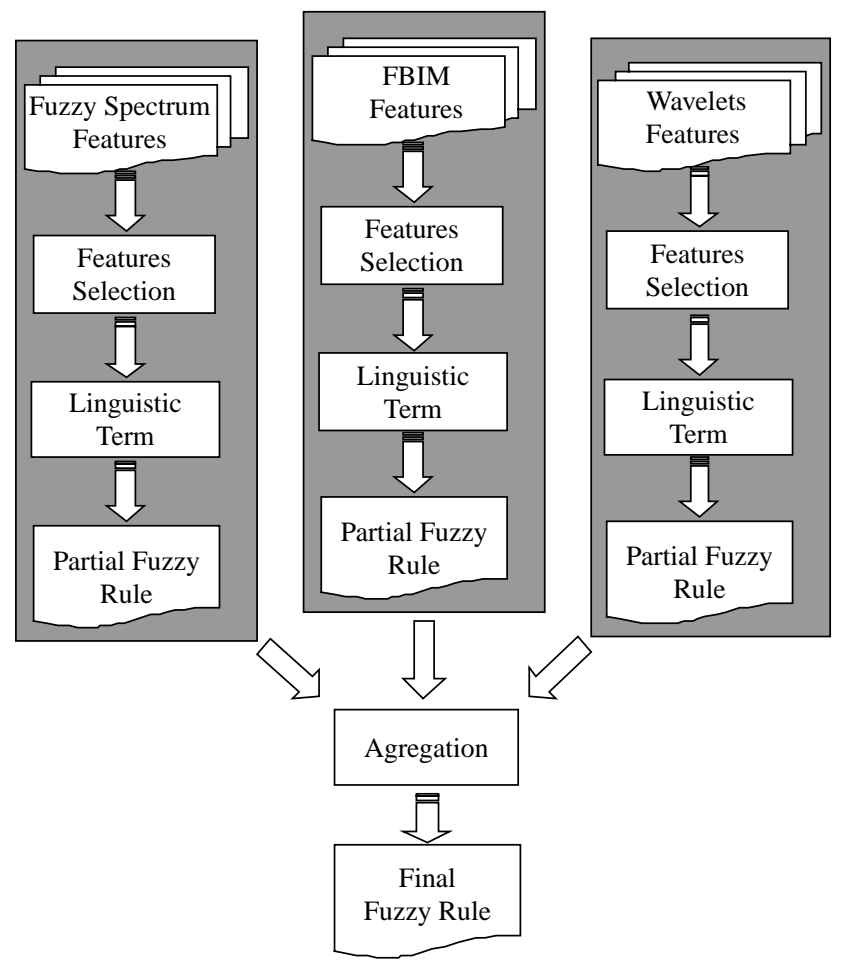

FWD22CBANV the result will be HistVar:6\| HistVar:7\# FWD22CBANV.

\section{System evaluation}

The system was evaluated and tested using a universe of 727 images $(128 \times 128$ pixels $)$ of 30 texture samples, from fabric, cork, and wood Portuguese industrial sectors and also from Brodatz database.

Figures 13-15 present some results of the system application in order to demonstrate its performance. Figures 13 and 14 show graphically the magnitude of the fuzzy rule response for each window under analysis. These graphics exemplify how the rule with the higher value defines the class of the texture that the window belongs. In both cases, the classes are clearly discriminated as response values are well apart.

Figure 15 shows the performance of the prototype for a subset of textures from the database (Figure 15(a)). Figure 15(b)-(e) show the response of each texture rule (gray bars) as well as the overall response of the rule that characterize the other textures (red bars), for the partial FBIM fuzzy rule, partial wavelet fuzzy rule, partial fuzzy spectrum fuzzy rule and global fuzzy rule, respectively. Higher mean response values are obtained in aggregation conditions. Concerning the partial rules, the wavelets present higher mean response values and lower difference, whereas FBIM allows a better discrimination between rules.

A specific advantage of the developed approach is that when a texture is presented to the inference system it gives a response with high value (normally higher than 0.85 ) for the rule that describes this texture. In contrast, the rules corresponding to the other textures give low response values. 
Figure 12 Membership degree of linguistic terms: (a) primitive FWD00M; (b) primitive FWD21CBNNV; (c) primitive FWD22CBNNV; (d) primitive FWD22CBANV

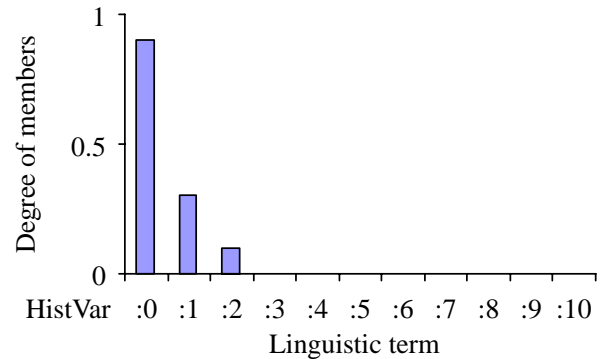

(a)

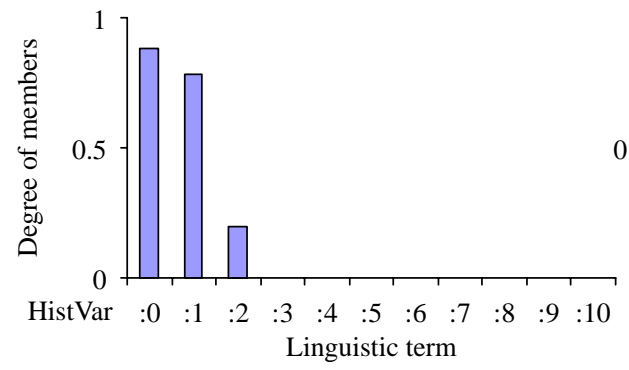

(c)

Figure 13 Response values of the rules, generated for BASE and UPPER texture, for each window under analysis

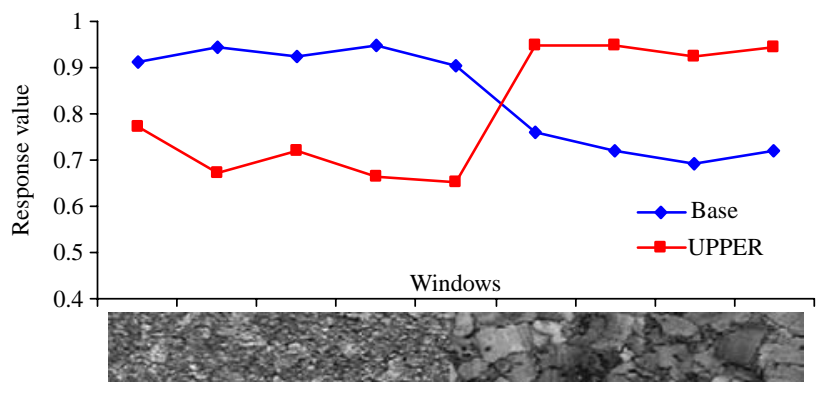

Figure 14 Response values of the rules, generated for MOTIVE and UPPER texture, for each window under analysis

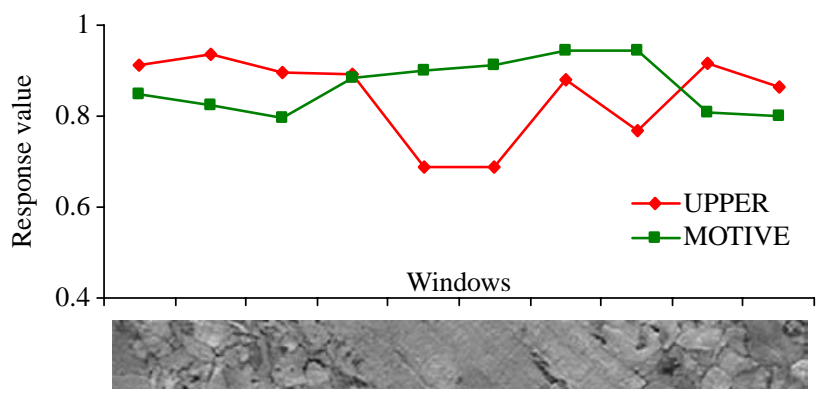

This means that the system creates disjoints rules and assures a good classification.

Considering the universe of samples under test, high classification rates were achieved. Specifically, 94 percent for wavelet partial fuzzy rule, 91 percent for FBIM partial fuzzy rule, 90 percent for fuzzy spectrum partial fuzzy rule, and 98 percent for the aggregated fuzzy rule.

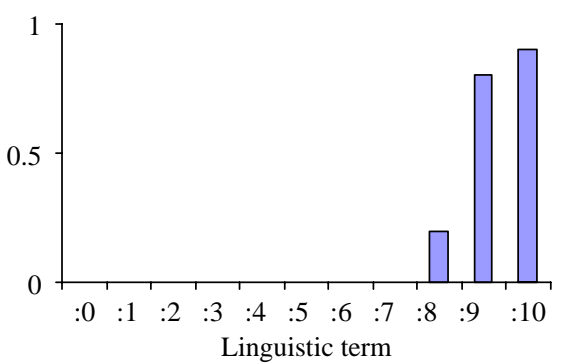

(b)

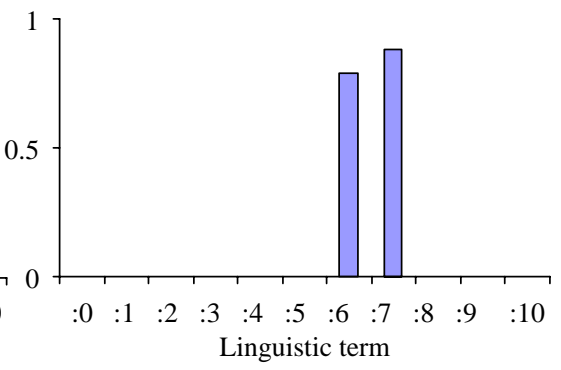

(d)

Figure 16 shows the application to a fabric image with a texture defect. For this sector, a 92 percent rate were achieved during fabric inspection procedures. The system was also applied to a cork inspection machine in an industrial environment, using only the FVW. It was used an image size of $1,024 \times 1,024$ pixels - pulnix camera, a matrox acquisition board, and it was developed in $\mathrm{C}++$. Good classification rates, higher than 90 percent, were achieved suggesting the efficiency of the inference system when applied to this Portuguese industrial sector.

\section{Conclusion}

In this paper, a generic prototype for texture segmentation was presented. In order to achieve higher flexibility, concerning the diversity of textures and its number, a fuzzy grammar inference system was used and several features from different techniques were aggregated to obtain the texture characterization.

The texture segmentation was based on six features extracted for each detail image of the wavelet transform of a gray scale image; and on two vectors, Z1r and Zsr extracted from the FBIM technique; and on 16 elements of the fuzzy spectrum.

Another advantage of the proposed solution is that the learning phase can be done with a unique sample of each type of texture.

High classification rates were achieved for inspection applications independently of the texture nature, suggesting the efficiency of the prototype when applied to different industrial sectors. The cork inspection as well as the fabric are important demanding sectors concerning the need of technologies for quality control, in the Portuguese industrial scenario. The prototype was tested in both sectors revealing good performance results, with classification rates higher than 90 percent. 
Figure 15 (a) Some examples of the textures used to texture segmentation procedure evaluation. Rule response for the images of (a); (b) partial FBIM; (c) partial wavelet; (d) partial fuzzy spectrum; (e) global

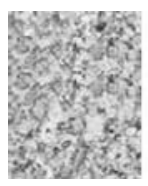

T1

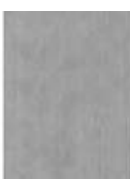

$\mathrm{T} 2$

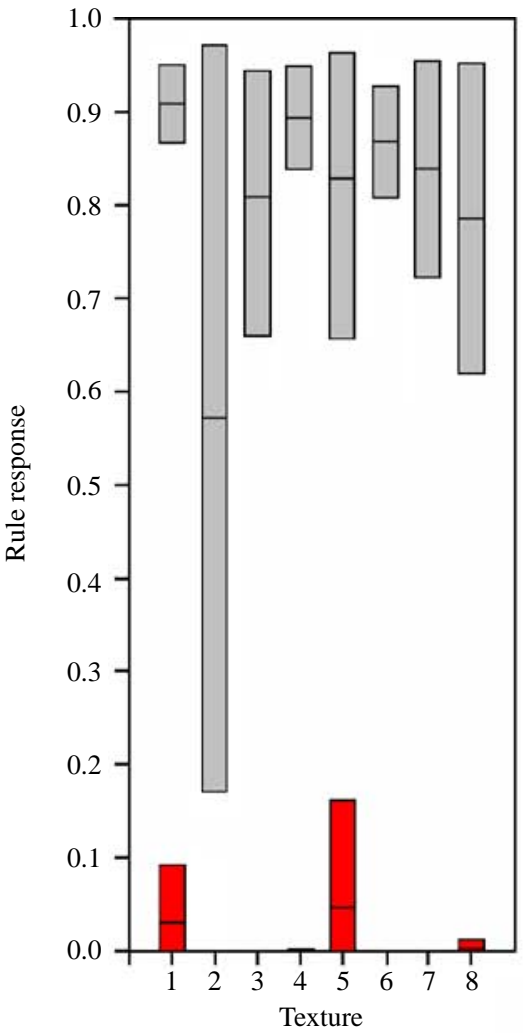

(b)
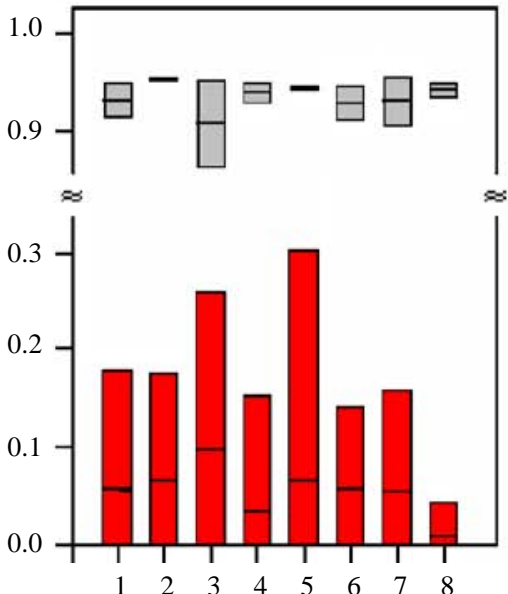

(c)

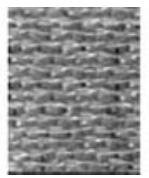

T4

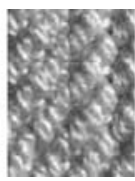

T5

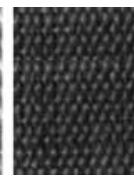

T6

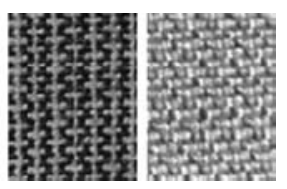

T7

T8

(a)

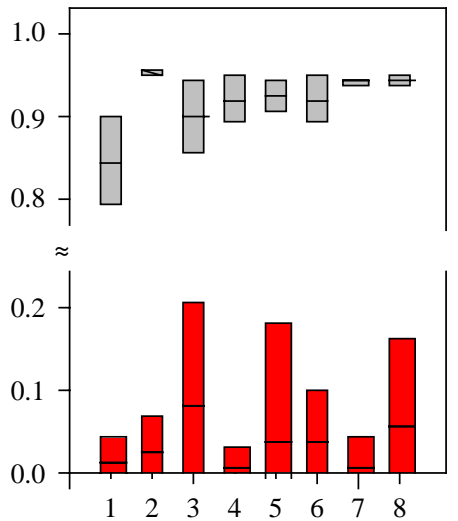

(d)

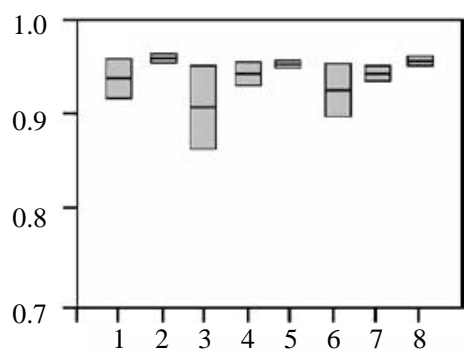

(e)

Rule response for the rule that characterizes the corresponding texture:

Maximum response value
Mean response value
Minimum response value

Responsible of the other values

Maximum response value

Mean response value

Minimum response value 
Figure 16 Application to fabric inspection: (a) original image; (b) segmentation result. $\mathrm{d} x=D$ and $\mathrm{d} y=D$

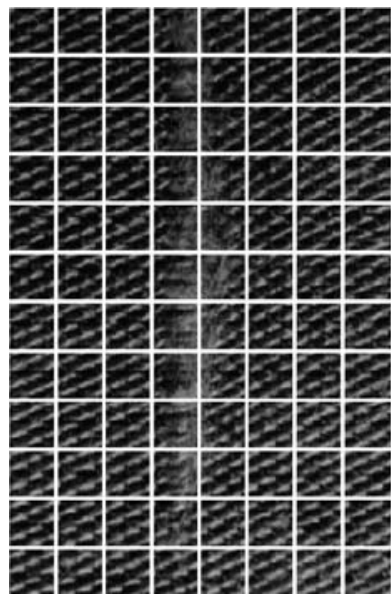

(a)

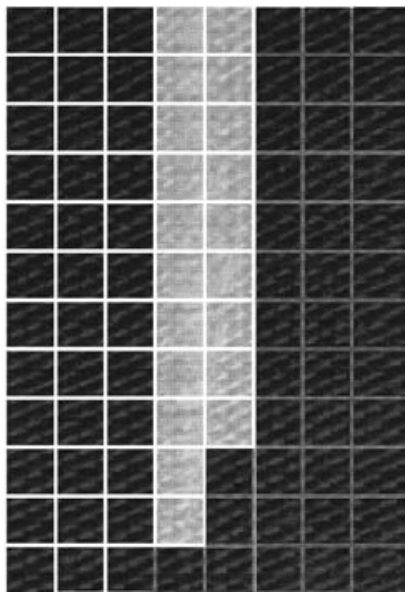

(b)
The flexibility of the solution was reinforced through its application in the development of a robot tracking system with a wavelet decomposition of three levels and window size of $45 \times 34$ pixels. In this case, results were also prominent allowing the achievement of a good tracking performance and a processing time of $40 \mathrm{~ms}$.

\section{References}

Ballard, D.H. and Brown, C.M. (1982), Computer Vision, Prentice-Hall, Englewood Cliffs, NJ.

Benedetto, J.J. and Frazier, M.W. (1994), Wavelets Mathematics and Applications, CRC Press, Boca Raton, FL.

Bennamoun, M. and Mamic, G.J. (2002), "Object recognition - fundamentals and case studies", in Singh, S. (Ed.), Advances in Pattern Recognition Series, SpringerVerlag, New York, NY.

Bezdek, J.C. and Pal, S.K. (1992), Fuzzy Models for Pattern Recognition, IEEE Press, New York, NY.

Bharati, H.M., Liu, J.J. and MacGregor, J.F. (2004), "Image texture analysis: methods and comparisons", Chemometrics and Intelligent Laboratory Systems, Vol. 72, pp. 57-71.

Bovik, A.C., Clark, M. and Geisler, W.S. (1990), "Multichannel texture analysis using localized spatial filters", IEEE Transactions on Pattern Analysis and Machine Intelligence, Vol. 12 No. 1, pp. 55-73.

Brodatz, P. (1966), "A Photographic album for artists and designers”, Dover, New York, NY, available at: www.ux.his. no/ tranden/brodatz.html (accessed February 3, 2004).

Bumble-Bee (2008), "Parser generator manual [online]", Bumble-Bee Software, available at: www. bumblebeesoftware.com (accessed August 30, 2008).

Carpenter, G.A. and Grossberg, S. (1992), Neural Networks for Vision and Image Processing, MIT Press, Cambridge, MA.

Chaudhuri, B.B. and Sarkar, N. (1995), "Texture segmentation using fractal dimension", IEEE Transactions on Pattern Analysis and Machine Intelligence, Vol. 17 No. 1, pp. 72-6.

Chen, C. and Chu, H. (2005), "Similarity measurement between images", Proceedings of the 29th Annual International Computer Software and Applications Conference, Edinburgh, Scotland, fuly 26-28, Vol. 2, pp. 41-2.
Chetverikov, D. (1995), "Texture anisotropy, symmetry, regularity: recovering structure and orientation from interaction maps", Proceedings 6th British Machine Vision Conference, Birmingham, UK, pp. 57-66.

Chetverikov, D. (1999), "Texture analysis using feature based pairwise interaction maps", Pattern Recognition, Vol. 32, pp. 487-502 (special issue on color and texture).

Clausi, D.A. and Yue, B. (2004), "Comparing coocurrence probabilities and Markov random fields for texture analysis of SAR sea ice imagery", IEEE Transactions on Geoscience and Remote Sensing, Vol. 42 No. 1, pp. 215-28.

Costa, L.F. and Cesar, R.M. Jr (2001), "Shape analysis and classification”, in Laplante, P.A. (Ed.), Image Processing Series, CRC Press, Boca Raton, FL.

Cross, G.R. and Jain, A.K. (1983), "Markov random field texture models", IEEE Transactions on Pattern Analysis and Machine Intelligence, Vol. PAMI-5 No. 1, pp. 25-39.

Cui, J., Wang, Y., Tan, T., Ma, L. and Sun, Z. (2004), "A fast and robust iris localization method based on texture segmentation", in Jain, A.K. and Ratha, N.K. (Eds), Proceedings of SPIE Biometric Technology for Human Identification, Orlando, USA, April 12, Vol. 5404, pp. 401-8.

Ducher, S.P., Nanyan, P., Lemineur, G., Benhamou, L. and Courteix, D. (2004), "Fractal analysis of bone texture: a screening tool for stress fracture risk", European fournal of Clinical Investigation, Vol. 34, pp. 137-42.

Duda, R.O. and Hart, P.E. (1973), Pattern Classification and Scene Analysis, Wiley, New York, NY.

Fan, G. and Xia, X. (2003), "Wavelet-based texture analysis and synthesis using hidden Markov models", IEEE Transactions on Circuits and Systems, Vol. 50 No. 1, pp. 106-20.

Gonzalez, R.C. and Woods, R.E. (1992), Digital Image Processing, Prentice-Hall, Englewood Cliffs, NJ.

Haralick, R.M. and Shapiro, L.G. (1992), Computer and Robot Vision, Vol. 1, Addison-Wesley, Reading, MA.

Heeger, D.J. and Bergen, J.R. (1995), "Pyramid-based texture analysis/synthesis", Proceedings of the 22nd Annual Conference on Computer Graphics and Interactive Techniques, Los Angeles, CA, USA, August 6-11, pp. 229-38.

Huang, Z., Zheng, C., Du, J. and Wan, Y. (2006), "Bark classification based on textural features using artificial neural networks", Advances in Neural Networks, Vol. 3972, pp. 355-60 (Lecture Notes in Computer Science).

Ivancic, F. and Malaviya, A. (1998), "An automatic rule base generation method for fuzzy pattern recognition with multiphased clustering", Proceedings of IEEE Conference of Knowledge Engineering System, Adelaide, Australia, April 21-23, Vol. 3, pp. 66-75.

Jafari-Khouzani, K. and Soltanian-Zadeh, H. (2005), "Radon transform orientation estimation for rotation invariant texture analysis", IEEE Transactions on Pattern Analysis and Machine Intelligence, Vol. 27 No. 6, pp. 1004-8.

Jang, J.R., Sun, C.T. and Mizutani, E. (1997), Neuro-fuzzy and Soft Computing, a Computational Approach to Learning and Machine Intelligence, Prentice-Hall, Englewood Cliffs, NJ.

Karkanis, S.A., Iakovidis, D.K., Maroulis, D.E., Karras, D.A. and Tzivras, M. (2003), "Computer-aided tumor detection in endoscopic video using color wavelet features", IEEE Transactions on Information Technology in Biomedicine, Vol. 7 No. 3, pp. 141-52.

Laine, A. and Fan, J. (1993), “Texture classification by wavelet packet signatures", IEEE Transactions on Pattern Analysis and Machine Intelligence, Vol. 15 No. 11, pp. 1186-90. 
Latif-Amet, A., Ertuzun, A. and Erçm, A. (2000), "An efficient method for texture detection: sub-band domain co-occurrence matrices", Image and Vision Computing, Vol. 18, pp. 543-53.

Lee, Y., Lee, J. and Hsueh, Y. (1998), "Texture classification using fuzzy uncertainty texture spectrum", Neurocomputing, Vol. 20 No. 1, pp. 115-22.

Liapis, S., Sifakis, E. and Tziritas, G. (2004), "Colour and texture segmentation using wavelets frame analysis, deterministic relaxation, and fast marching algorithms", Fournal of Visual Communications and Image Representation, Vol. 15, pp. 1-26.

Liu, Y., Lin, W. and Hays, J. (2004), "Near-regular texture analysis and manipulation", ACM Transactions on Graphics (SIGGRAPH 2004), Vol. 23 No. 3, pp. 368-76, Los Angeles, CA, August 10-12.

Livens, S. (1998), "Image analysis for material characterization", $\mathrm{PhD}$ thesis, Vol. 1,2, Universitaire Instelling Antwerpen, Antwerpen.

Looney, C.G. (2002), "Pattern recognition", in Cho, Hyngsuck (Ed.), Opto-Mechatronic Systems Handbook: Technical Applications, Handbook Series for Mechanical Engineering, Vol. 10, CRC Press, Boca Raton, FL.

Ma, L., Tan, T., Wang, Y. and Zhang, D. (2003), "Personal identification based on iris texture analysis", IEEE Transactions on Pattern Analysis and Machine Intelligence, Vol. 25 No. 12, pp. 1519-33.

Malaviya, A. (1996), “On-line handwriting recognition with a fuzzy feature description language”, $\mathrm{PhD}$ thesis, Technische Universitat Berlin, Berlin.

Mirmehdi, M., Marik, R., Petrou, M. and Kittler, J. (1996), "Iterative morphology for fault detection in stochastic texture", IEEE Electronic Letters, Vol. 32 No. 5, pp. 443-4.

Moraes, R.M. (1996), "Image classification using mathematical morphology", Proceedings of SIBGRAPI9, Caxambu, Brazil, pp. 357-8.

Munzenmayer, C., Wilharm, S., Hornegger, J. and Wittenberg, T. (2005), "Illumination invariant color texture analysis based on sum and difference histograms", Pattern Recognition, Vol. 3663, pp. 17-24 (Lectures Notes in Computer Science).

Neary, D. (2000), "Fractal methods in image analysis and coding", MSc thesis, Dublin City University, Dublin.

Paragios, N. and Deriche, R. (2002), "Geodesic active regions level set methods for supervised texture segmentation", International fournal of Computer Vision, Vol. 46 No. 3, pp. 223-47.

Perry, S.W., Wong, H.S. and Guan, L. (2002), "Adaptive image processing - a computational intelligence perspective", in Laplante, P.A. (Ed.), Image Processing Series, CRC Press, Boca Raton, FL.

Prats-Montalban, J.M. and Ferrer, A. (2007), "Integration of colour and textural information in multivariate image analysis: defect detection and classification issues", fournal of Chemometrics, Vol. 21, pp. 10-23.

Pratt, W.K. (2001), Digital Image Processing, 3rd ed., Wiley, New York, NY.
Randen, T. (1997), "Filter and filter bank design for image texture recognition", $\mathrm{PhD}$ thesis, Stavanger College, University of Science and Technology, Stavanger.

Russ, J.C. (1995), The Image Processing Handbook, 2nd ed., CRC Press, Boca Raton, FL.

Tay, P. and DeBrunner, V. (2002), "Discrete wavelet transform with optimal joint localization for determining the number of image texture segments", Proceedings of International Conference on Image Processing, Rochester, NY, USA, September 22-25, Vol. 3, pp. 281-4.

Teuner, A., Pichler, O. and Hosticka, B.J. (1995), "Unsupervised texture segmentation of images using tuned matched Gabor filters", IEEE transactions on image processing, Vol. 4 No. 6, pp. 863-70.

Tsai, D. and Huang, T. (2003), "Automated surface inspection for statistical textures", Image and Vision Computing, Vol. 21, pp. 307-23.

Tseng, D.C. and Lai, C.C. (1999), "A genetic algorithm for MRF-based segmentation of multi-spectral textured images", Pattern Recognition Letters, Vol. 20, pp. 1499-510.

Tzanakou, E.M. (2000), "Supervised and unsupervised pattern recognition: feature extraction and computational intelligence", in David Irwin, J. (Ed.), Industrial Electronics Series, CRC Press, Boca Raton, FL.

Unser, M. (1995), "Texture classification and segmentation using wavelet frames", IEEE Transactions on Image Processing, Vol. 4 No. 11, pp. 1549-60.

VisTEX (1997), "Texture gallery database", available at: www-white.media.mit.edu/vismod/imagery/VisionTexture/ (accessed February 3, 2004)

Williams, P.S. (1999), “The automatic hierarchical decomposition of images into sub-images for use in image recognition and classification", $\mathrm{PhD}$ thesis, Department of Electrical and Electronic Engineering, University of Western Australia, Perth.

Wouwer, G.V. (1998), "Wavelets for multiscale texture analysis", $\mathrm{PhD}$ thesis, Universitaire Instelling Antwerpen, Antwerpen.

Wouwer, G.V., Scheunders, P. and Dyck, D.V. (1998), "Statistical texture characterization from discrete wavelet representations", IEEE Transactions on Image Processing, Vol. 8 No. 4, pp. 592-8.

Yager, R.R. and Zadeh, L.A. (1992), An Introduction to Fuzzy Logic Applications in Intelligent Systems, Kluwer Academic Press, Boston, MA.

Zhang, J. and Tan, T. (2002), "Brief review of invariant texture analysis methods", Pattern Recognition, Vol. 35, pp. 735-47.

Zhang, A., Cheng, B. and Acharya, R. (1995), “Texture-based image retrieval using fractal codes", Technical Report: TR/ 95-19, State University of New York, Buffalo, NY.

\section{Corresponding author}

Manuel Ferreira can be contacted at: mjf@dei.uminho.pt 